International Journal of Diabetology \& Vascular Disease Research (JJDVR)

ISSN:2328-353X

\title{
Maintaining Metabolically Healthy Overweight and Risk of Incident Type 2 Diabetes or Cardiovascular Disease
}

Research Article

Hashimoto $\mathrm{Y}^{1}$, Tanaka $\mathrm{M}^{1}$, Senmaru $\mathrm{T}^{1}$, Asano $\mathrm{M}^{1}$, Yamazaki $\mathrm{M}^{1}$, Oda $\mathrm{Y}^{1}$, Toda $\mathrm{H}^{2}$, Nakamura $\mathrm{N}^{1}$, Fukui $^{1 *}$

Department of Endocrinology and Metabolism, Kyoto Prefectural University of Medicine, Graduate School of Medical Science, Kyoto, Japan.

Department of Internal Medicine, Oike Clinic, Kyoto, Japan.

\begin{abstract}
Background: Recent studies reveal that metabolically healthy obesity, which is free from the metabolic complications of obesity at present, is a risk of incident diabetes or cardiovascular disease (CVD). It may be because many metabolically healthy obesity individuals transit to metabolically abnormal obesity over time. However, the association between maintaining metabolically healthy obesity and incident diabetes or CVD is still unclear.

Methods: 2190 participants without diabetes and/or CVD were examined at baseline and at 5-year follow-up, using a retrospective cohort study design. Metabolically abnormal was defined as having $\geq 2$ of metabolic abnormalities, hypertension, hypertriglyceridemia, low HDL cholesterol and impaired fasting glucose. Overweight was defined as BMI $\geq 23 \mathrm{~kg} /$ $\mathrm{m}^{2}$, which is recommended by WHO for Asian. Adjusted odds ratio (OR) and $95 \%$ confidence interval (CI) for incident diabetes or CVD at follow-up were estimated adjusting for potential confounders, compared to maintaining metabolically healthy non-overweight (MHNO).

Results: At the follow-up examination, 82 participants developed diabetes. Crude incident proportions of diabetes were $0.6 \%$ in maintained MHNO (case $/ \mathrm{N}=5 / 859)$ and $1.3 \%$ in maintained metabolically healthy overweight $(\mathrm{MHO})(4 / 314)$. Adjusted OR for incident diabetes of maintaining $\mathrm{MHO}$ was 1.71 (95\% CI 0.42-6.57, $P=0.438)$. At the follow-up examination, 51 participants developed CVD. Crude incident proportions of CVD were $0.8 \%$ in maintained MHNO $(7 / 859)$ and $1.6 \%$ in maintained MHO (5/314). Adjusted OR for incident CVD of maintaining MHO was 1.42 (95\% CI 0.41-4.56, $P$ $=0.565)$.

Conclusions: Maintaining MHO phenotype was not associated with higher risk of incident diabetes or CVD.
\end{abstract}

Keywords: Obesity; Body Mass Index; Type 2 Diabetes; Metabolic Syndrome; Epidemiology; Cardiovascular Disease.

Abbreviations: FPG: Fasting Plasma Glucose, HDL: High-Density Lipoprotein, BMI: Body Mass Index, BP: Blood Pressure, MHNO: Metabolically Healthy Non-Overweight, MHO: Metabolically Healthy Overweight, MANO: Metabolically Abnormal Non-Overweight, MAO: Metabolically Abnormal Overweight.

\section{*Corresponding Author:}

Michiaki Fukui M.D. Ph.D,

Department of Endocrinology and Metabolism

Kyoto Prefectural University of Medicine, Graduate School of Medical Science, 465 Kajii-cho, Kawaramachi-Hirokoji, Kamigyo-ku, Kyoto 6028566, Japan.

Tel: +81-75-251-5505

Fax: +81-75-252-3721

E-mail: sayarinapm@hotmail.com

Received: August 13, 2015

Accepted: September 24, 2015

Published: September 25, 2015

Citation: Fukui M et al., (2015) Maintaining Metabolically Healthy Overweight and Risk of Incident Type 2 Diabetes or Cardiovascular Disease. Int J Diabetol Vasc Dis Res, S3:001 1-6. doi: http://dx.doi. org $/ 10.19070 / 2328-353 X-S I 03001$

Copyright: Fukui $\mathbf{M}^{\circ}$ 2015. This is an open-access article distributed under the terms of the Creative Commons Attribution License, which permits unrestricted use, distribution and reproduction in any medium, provided the original author and source are credited.

\section{Introduction}

Obesity [1] and metabolic syndrome [2] are major public health problems worldwide and frequently co-exist. Recent studies have identified a subset of obese individuals who have a low burden of adiposity-related metabolic abnormalities compared with at risk obese individuals, the so-called metabolically healthy obesity phenotype [3-5]. Metabolically healthy obesity phenotype is characterized by high levels of insulin sensitivity, low prevalence of hypertension and a favorable fasting glucose, lipid, and inflammation profile, compared with metabolically abnormal obesity phenotype [6]. In fact, we previously reported that not metabolically healthy obesity but metabolically abnormal obesity phenotype was associated with incident chronic kidney disease [7].

However, recent studies revealed that metabolically healthy obesity phenotype was also a risk of incident Type 2 diabetes [8-10] and cardiovascular disease (CVD) [10-12]. On the other hand, among the metabolically healthy obesity individuals, about half of them transit to metabolically abnormal obesity phenotype over time $[7,13,14]$. Thus, there is a possibility that metabolically healthy obesity individuals, who developed Type 2 diabetes or CVD, transitioned to metabolically abnormal obesity phenotype before incident Type 2 diabetes or CVD. However, evidence of whether 
maintaining metabolically healthy overweight (MHO) phenotype is associated with incident Type 2 diabetes or CVD is currently limited. Only a few studies have shown data on the incidence rate of Type 2 diabetes in relation to maintaining metabolically healthy obesity over time $[15,16]$. Thus, it is still controversial whether maintaining $\mathrm{MHO}$ phenotype is associated with incident Type 2 diabetes. In addition, it is still unclear whether maintaining $\mathrm{MHO}$ phenotype is associated with incident CVD. Therefore, the purpose of this cohort study was to investigate whether maintaining MHO phenotype was associated with incident Type 2 diabetes or CVD.

\section{Methods}

\section{Study Participants and Study Design}

The Oike Health Survey is an ongoing cohort investigation of risk factors for chronic diseases including hypertension, diabetes and chronic kidney disease. The Oike Clinic (Kyoto, Japan) provides regular health check-up for the employees of various companies. In Japan, yearly routine examination for employees is legally mandated and all or most of the costs for the health checkup are usually paid by their employers.

In this retrospective cohort study, we enrolled 2522 participants who received health check-up examinations at Oike Clinic both in 2009 and in 2014. We excluded participants with missing data of covariates and participants who had Type 2 diabetes and/or CVD at baseline examination which was performed in 2009. The Ethical Committee of the Oike Clinic approved this study and the study was conducted in accordance with Declaration of Helsinki. Each participant provided informed consent.

\section{Data Collection and Measurements}

All participants provided details of their demographics. Participants were classified as non-smokers, ex-smokers or current smokers according to a self-administered questionnaire. Habit of alcohol was defined as daily alcohol consumption. Regular exercise was defined as performing any kind of sports at least once a week. Body mass index (BMI) was calculated as weight in kilograms divided by height in meters squared. After an overnight fast, venous blood was collected for the measurement of the levels of various factors, including fasting plasma glucose (FPG), triglycerides and high-density lipoprotein (HDL) cholesterol. $\mathrm{HbA1c}$ was assayed using high-performance liquid chromatography. The value for HbA1c (\%) was estimated as the National Glycohemoglobin Standardization Program value (\%) calculated by the formula HbA1c $(\%)=$ HbA1c (Japan Diabetes Society) $(\%) \times 1.02+0.25 \%$ [17]. Diagnosis of Type 2 diabetes was made according to the American Diabetes Association (ADA) criteria of a FPG level of $\geq 7.0 \mathrm{mmol} / 1$, self-reported clinician-diagnosed diabetes or $\mathrm{HbA} 1 \mathrm{c} \geq 6.5 \%$ [18]. The incidence of cardiovascular events among the study participants was assessed by a self-administered questionnaire when they visited the center for their health check-ups. Cardiovascular disease was defined as unstable angina, acute myocardial infarction, silent myocardial infarction, ischemic stroke or cerebral hemorrhage.

\section{Definitions of Metabolic Phenotypes}

We used a cut-off point of body mass index (BMI) $23 \mathrm{~kg} / \mathrm{m}^{2}$ to define overweight $\left(\geq 23.0 \mathrm{~kg} / \mathrm{m}^{2}\right)$ or non-overweight $(<23.0 \mathrm{~kg} /$ $\mathrm{m}^{2}$ ) according to the criteria proposed by the Regional Office for the Western Pacific Region of WHO (WPRO), the International Association for the Study of Obesity and the International Obesity Task Force [19]. The validity of definition was confirmed previously $[20,21]$. In addition, the ADA also recommended a cut-off point of BMI 23 for the Asian American population, because this population was susceptible to overweight on incident Type 2 diabetes [22]. We used four metabolic factors (hypertension, hypertriglyceridemia, low HDL-cholesterol concentration and impaired fasting glucose), which were defined by International Diabetes Federation (IDF) [23], to determine whether the participant was metabolically healthy or metabolically abnormal. Participants with a systolic blood pressure $(\mathrm{BP}) \geq 130 \mathrm{~mm} \mathrm{Hg}$ and/or a diastolic $\mathrm{BP} \geq 85 \mathrm{~mm} \mathrm{Hg}$ or who were under medical treatment were considered to have hypertension. Elevated triglycerides was indicated by $\geq 1.70 \mathrm{mmol} / \mathrm{L}$ or treatment of hyperlipidemia and reduced HDL-cholesterol was indicated by $<1.03 \mathrm{mmol} / \mathrm{L}$ in men and $<$ $1.29 \mathrm{mmol} / \mathrm{L}$ in women. Participants with FPG $\geq 5.6 \mathrm{mmol} / \mathrm{L}$ or who were under medical treatment were consider to impaired fasting glucose. A metabolically healthy state was considered if none or 1 of the metabolic factors based on the IDF definition was present and a metabolically abnormal state was declared if 2 or more metabolic factors were present [23]. The validity of this definition was confirmed previously [7]. Then, participants were categorized into 4 phenotypes: 1) metabolically healthy non-overweight (MHNO), 2) metabolically healthy overweight (MHO), 3) metabolically abnormal non-overweight (MANO) or 4) metabolically abnormal overweight (MAO) at examinations in 2009 and in 2014. Then we categorized participants into 16 groups based on combinations of the four phenotypes at examinations in 2009 and those in 2014.

\section{Statistical Analysis}

Continuous variables were expressed as mean \pm standard deviation and categorical variables were expressed as number (percent). The differences of continuous or categorical variables among four phenotypes was determined by Tukey HSD (honestly significant difference) test and $\chi^{2}$ test. We performed logistic regression analyses to assess the association of combinations of the four phenotypes at examinations in 2009 and in 2014 with incident Type 2 diabetes, adjusting for covariates, including age, sex, smoking status, habit of alcohol, regular exercise and family history of diabetes. Then, we also performed logistic regression analysis to assess the association of combinations of the four phenotypes at examinations in 2009 and in 2014 with incident CVD, adjusting for covariates, including age, sex, smoking status, habit of alcohol and regular exercise. The statistical analyses were performed using the JMP software version 10.0 software (SAS Institute Inc., Cary, North Carolina) and $P$ value $<0.05$ was considered to represent a statistically significant difference.

\section{Results}

In 2009, we enrolled 2522 participants (1521 men and 1001 women) (Figure 1). Among them, 78 participants (53 men and 25 women) were excluded because of missing data and 254 participants (208 men and 46 women) were excluded because of Type 2 diabetes and/or CVD at baseline examination. Thus, the study population consisted of 2190 participants (1260 men and 930 women).

The characteristics of participants were shown in Table 1. The 
Figure 1. Inclusion and exclusion flow chart.

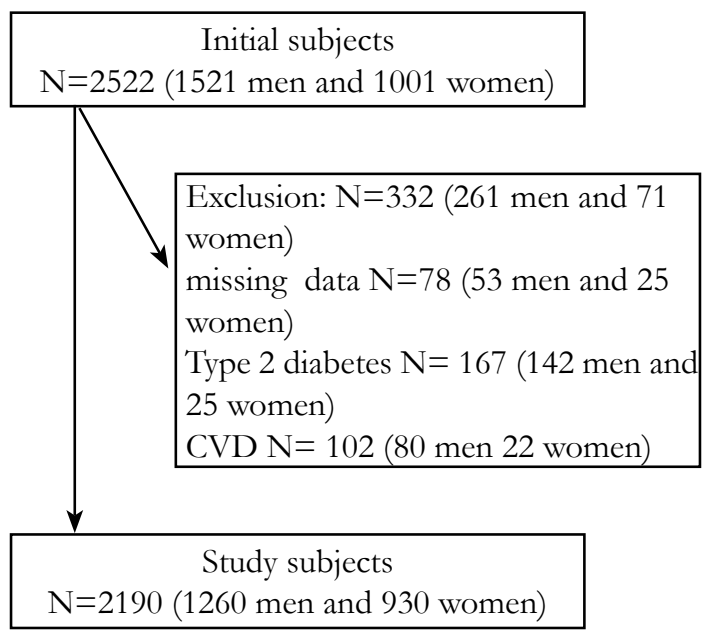

Table 1. Characteristics of study participants.

\begin{tabular}{|c|c|c|c|c|}
\hline & MHNO & MHO & MANO & MAO \\
\hline $\mathrm{N}$ & 1110 & 550 & 184 & 346 \\
\hline Age (years) & $52.4 \pm 9.2$ & $53.0 \pm 10.1$ & $59.4 \pm 9.2 * \dagger$ & $58.3 \pm 9.8^{*}+$ \\
\hline Male $\int$ & $474(42.7 \%)$ & $402(73.1 \%)$ & $107(58.2 \%)$ & $277(80.1 \%)$ \\
\hline $\mathrm{BMI}\left(\mathrm{kg} / \mathrm{m}^{2}\right)$ & $20.4 \pm 1.7$ & $25.1 \pm 1.9^{*}$ & $21.2 \pm 1.5^{*}+$ & $25.9 \pm 2.3^{*}+\neq$ \\
\hline Waist circumstance $(\mathrm{cm})$ & $74.6 \pm 6.3$ & $86.8 \pm 6.4^{*}$ & $78.6 \pm 5.9 * \dagger$ & $89.9 \pm 6.2^{*}+\ddagger$ \\
\hline Systolic BP (mmHg) & $113.5 \pm 13.6$ & $123.0 \pm 12.7 *$ & $129.5 \pm 12.4 * \dagger$ & $134.0 \pm 13.0^{*} \dagger \ddagger$ \\
\hline Diastolic BP $(\mathrm{mmHg})$ & $70.9 \pm 9.7$ & $77.6 \pm 8.9^{*}$ & $81.0 \pm 8.8^{*} \dagger$ & $83.9 \pm 8.6^{*}+\neq$ \\
\hline $\begin{array}{c}\text { Fasting plasma glucose } \\
(\mathrm{mmol} / \mathrm{L})\end{array}$ & $4.9 \pm 0.4$ & $5.1 \pm 0.4^{*}$ & $5.4 \pm 0.6^{*} \dagger$ & $5.5 \pm 0.5^{*} \dagger$ \\
\hline HbA1c $(\%)$ & $5.3 \pm 0.3$ & $5.3 \pm 0.4$ & $5.5 \pm 0.3^{*} \dagger$ & $5.6 \pm 0.4^{*} \dagger$ \\
\hline Total cholesterol (mmol/L) & $5.4 \pm 0.8$ & $5.4 \pm 0.8$ & $5.4 \pm 0.9$ & $5.3 \pm 0.8$ \\
\hline Triglycerides $(\mathrm{mmol} / \mathrm{L})$ & $0.9 \pm 0.5$ & $1.2 \pm 0.5^{*}$ & $1.6 \pm 1.0^{*} \dagger$ & $1.9 \pm 1.2^{*}+\ddagger$ \\
\hline HDL cholesterol $(\mathrm{mmol} / \mathrm{L})$ & $1.9 \pm 0.4$ & $1.6 \pm 0.4^{*}$ & $1.6 \pm 0.5^{*}$ & $1.4 \pm 0.4 * \dagger \neq$ \\
\hline \multicolumn{5}{|l|}{ Smoking status $\int$} \\
\hline Past smoker & $95(8.5 \%)$ & $98(17.8 \%)$ & $24(13.0 \%)$ & $59(16.4 \%)$ \\
\hline Current smoker & $150(13.5 \%)$ & $99(18.0 \%)$ & $24(13.0 \%)$ & $64(18.5 \%)$ \\
\hline Regular exercise & $654(58.9 \%)$ & $339(61.6 \%)$ & $117(63.6 \%)$ & $218(63.0 \%)$ \\
\hline Habit of alcohol $\sqrt{ }$ & $355(32.0 \%)$ & $219(39.9 \%)$ & $76(41.3 \%)$ & $151(43.6 \%)$ \\
\hline Family history of diabetes & $192(17.3 \%)$ & $105(19.1 \%)$ & $40(21.7 \%)$ & $80(23.1 \%)$ \\
\hline \multicolumn{5}{|l|}{$\begin{array}{l}\text { Metabolic phenotype of } \\
\text { the follow-up examination }\end{array}$} \\
\hline MHNO & $859(77.4 \%)$ & $57(10.4 \%)$ & $41(22.3 \%)$ & $9(2.6 \%)$ \\
\hline $\mathrm{MHO}$ & $83(7.5 \%)$ & $314(57.1 \%)$ & $9(4.9 \%)$ & $64(18.5 \%)$ \\
\hline MANW & $138(12.4 \%)$ & $12(2.2 \%)$ & $116(63.0 \%)$ & $25(7.2 \%)$ \\
\hline MAO & $30(2.7 \%)$ & $167(30.4 \%)$ & $18(9.8 \%)$ & $248(71.7 \%)$ \\
\hline
\end{tabular}

MHNO; Metabolically healthy non-overweight, MHO; Metabolically healthy overweight, MANO; Metabolically abnormal nonoverweight, MAO; Metabolically abnormal overweight. Habit of exercise was defined as performing any kind of sports at least once a week. Habit of alcohol was defined as daily alcohol consumption. Data are number (\%) or mean \pm standard deviation. The analyses of continuous variables among four groups were performed by Tukey HSD test. $* P<0.05$ vs. MHNO, $\nmid P<0.05$ vs. MHO, $¥ P<0.05$ vs.

MANO. The analyses of categorical variables among four groups were performed by $\chi^{2}$ test. $\$ P<0.05$.

prevalence of MHNO, MHO, MANO and MAO at baseline examination were 50.7\% $(\mathrm{n}=1110), 25.1 \%(\mathrm{n}=550), 8.4 \%(\mathrm{n}=$ $184)$ and $15.8 \%(\mathrm{n}=346)$, respectively. At the follow-up examination, which was performed 5 years after baseline examination, among the participants who were $\mathrm{MHO}$ phenotype at baseline examination, $10.4 \%(\mathrm{n}=57 / 550)$ of them transitioned to MHNO, $57.1 \%(314 / 550)$ of them maintained MHO, 2.2\% (12/550) of them transitioned to MANO and 30.4\% $(n=167 / 550)$ of them transitioned to MAO phenotype.

\section{Incident Type 2 Diabetes}

At the follow-up examination, 82 participants developed Type 2 diabetes. Among the participants who were $\mathrm{MHO}$ phenotype at 
baseline examination, none of them who transitioned to $\mathrm{MHNO}$ $($ case $/ \mathrm{n}=0 / 57), 1.3 \%$ of them who maintained MHO (4/314), $8.3 \%$ of them who transitioned to MANO $(1 / 12)$ and $4.8 \%$ of them who transitioned to MAO (8/167) phenotype developed Type 2 diabetes at follow-up examination.

Odds ratios for incident Type 2 diabetes at 5 years after baseline examination according to combination of metabolic phenotypes at baseline and follow-up examinations are shown in Table 2. Compared with maintaining MHNO, the adjusted OR for incident Type 2 diabetes of maintaining MHO, transitioning from $\mathrm{MHO}$ to MANO and transitioning from MHO to MAO were 1.71 (95\% CI 0.42-6.57, $P=0.438), 17.5$ (95\% CI 0.87-123.6, $P=$ $0.058)$ and $6.78(95 \%$ CI 2.19-23.0, $P=0.001)$, respectively.

Incident Cardiovascular Disease
At the follow-up examination, 51 participants developed CVD. Among the participants who were MHO phenotype at baseline examination, $1.8 \%$ of them who transitioned to MHNO (case/n $=1 / 57), 1.6 \%$ of them who maintained MHO (5/314), $25 \%$ of them who transitioned to MANO (3/12) and $2.4 \%$ of them who transitioned to MAO (4/167) phenotype developed CVD at follow-up examination.

Odds ratios for incident cardiovascular disease at 5 years after baseline examination according to combination of metabolic phenotypes at baseline and follow-up examinations are shown in Table 3. Compared with maintaining MHNO, the adjusted OR for incident CVD of transitioning from $\mathrm{MHO}$ to $\mathrm{MHNO}$, maintaining $\mathrm{MHO}$, transitioning from $\mathrm{MHO}$ to MANO or transitioning from MHO to MAO were 1.50 (95\% CI 0.08-8.92, $P=0.720)$,

Table 2. Odds ratios for incident Type 2 diabetes at 5 years after baseline examination according to combination of metabolic phenotypes at baseline and follow-up examinations.

\begin{tabular}{|c|c|c|c|c|c|c|c|c|}
\hline & Case of diabetes & Model 1 & $\boldsymbol{P}$ value & Model 2 & Pvalue & Model 3 & $\boldsymbol{P}$ value \\
\hline \multicolumn{7}{|c|}{ Metabolic phenotype of the baseline and follow-up examinations (baseline-follow-up) } \\
\hline MHNO-MHNO & $5 / 859$ & 1 Reference) & - & 1 (Reference) & - & 1 (Reference) & - \\
\hline MHO-MHNO & $0 / 57$ & NEE & - & NEE & - & NEE & - \\
\hline MHO-MHO & $4 / 314$ & $2.20(0.54-8.38)$ & 0.253 & $1.85(0.45-7.12)$ & 0.373 & $1.71(0.42-6.57)$ & 0.438 \\
\hline MHO-MANO & $1 / 12$ & $15.5(0.78-107.5)$ & 0.066 & $13.6(0.68-95.5)$ & 0.077 & $17.5(0.87-123.6)$ & 0.058 \\
\hline MHO-MAO & $8 / 167$ & $8.59(2.83-28.7)$ & $<0.001$ & $7.21(2.33-24.5)$ & $<0.001$ & $6.78(2.19-23.0)$ & 0.001 \\
\hline
\end{tabular}

NEE; Not enough events to evaluate, MHNO; Metabolically healthy non-overweight, MHO; Metabolically healthy overweight, MANO; Metabolically abnormal non-overweight, MAO; Metabolically abnormal overweight. Ex- and current smoker were used with none smoker as a reference group. Model 1; unadjusted. Model 2; adjusted for age and sex. Model 3; adjusted for Model 2 plus habit of alcohol, habit of exercise, smoking status and family history of diabetes.

Table 3. Odds ratios for incident cardiovascular disease at 5 years after baseline examination according to combination of metabolic phenotypes at baseline and follow-up examinations.

\begin{tabular}{|c|c|c|c|c|c|c|c|c|}
\hline & Case of CVD & Model 1 & $\boldsymbol{P}_{\text {value }}$ & Model 2 & $\boldsymbol{P}_{\text {value }}$ & Model 3 & $\boldsymbol{P}_{\text {value }}$ \\
\hline \multicolumn{7}{|c|}{ Metabolic phenotype of the baseline and follow-up examinations (baseline-follow-up) } \\
\hline MHNO-MHNO & $7 / 859$ & 1 (Reference) & - & 1 (Reference) & - & 1 (Reference) & - \\
\hline MHO-MHNO & $1 / 57$ & $2.17(0.12-12.5)$ & 0.512 & $1.61(0.08-9.49)$ & 0.679 & $1.50(0.08-8.92)$ & 0.72 \\
\hline MHO-MHO & $5 / 314$ & $1.97(0.58-6.21)$ & 0.792 & $1.43(0.41-4.58)$ & 0.559 & $1.42(0.41-4.56)$ & 0.565 \\
\hline MHO-MANO & $3 / 12$ & $40.6(7.83-174.6)$ & $<0.001$ & $30.1(5.14-146.9)$ & $<0.001$ & $32.0(5.44-157.6)$ & $<0.001$ \\
\hline MHO-MAO & $4 / 167$ & $2.99(0.78-10.0)$ & 0.105 & $1.85(0.47-6.33)$ & 0.352 & $1.84(0.47-6.27)$ & 0.359 \\
\hline
\end{tabular}

CVD; cardiovascular disease, NEE; Not enough events to evaluate, MHNO; Metabolically healthy non-overweight, MHO; Metabolically healthy overweight, MANO; Metabolically abnormal non-overweight, MAO; Metabolically abnormal overweight. Ex- and current smoker were used with none smoker as a reference group. Model 1; unadjusted. Model 2; adjusted for age and sex. Model 3; adjusted for Model 2 plus habit of alcohol, habit of exercise and smoking status.

1.42 (95\% CI 0.41-4.56, $P=0.565), 32.0$ (95\% CI 5.44-157.6, $P<$ $0.001)$ and $1.84(95 \% \mathrm{CI} 0.47-6.27, P=0.359)$, respectively.

\section{Discussion}

The major finding of our study is that maintaining MHO phenotype was not associated with higher risk of incident Type 2 diabetes or CVD. On the other hand, transitioning from $\mathrm{MHO}$ to metabolically abnormal phenotype was associated with incident Type 2 diabetes. Furthermore, transitioning from MHO to
MANO was associated with incident CVD.

Recent studies reported that metabolically healthy obesity phenotype was a risk of incident Type 2 diabetes [8-10] and CVD [10$12]$. However, these studies only evaluated the metabolic phenotype of the baseline examination. Only a few studies have shown the association between maintaining metabolically healthy obesity phenotype and incident Type 2 diabetes $[15,16]$. Appleton et al.[15] showed that the individuals who maintained metabolically healthy obesity were not associated with incident Type 2 diabetes. 
On the other hand, Heianza et al. [16] showed that the individuals who maintained metabolically healthy obesity were associated with incident Type 2 diabetes. Thus, it has been unclear whether maintaining MHO phenotype is associated with incident Type 2 diabetes. In addition, it remains to be elucidated whether maintaining $\mathrm{MHO}$ phenotype is associated with incident CVD. Our study clarified that maintaining $\mathrm{MHO}$ phenotype was not associated with higher risk of incident Type 2 diabetes or CVD. On the other hand, transitioning from MHO to MANO was associated with incident Type 2 diabetes, although there was not statistically significance, and transitioning from $\mathrm{MHO}$ to MAO was associated with incident Type 2 diabetes. In addition, transitioning from MHO to MANO was associated with incident CVD. Thus, not obesity but metabolic abnormality might be important for incident Type 2 diabetes or CVD.

It is well known that overweight or obesity, which has close association with insulin resistance [24], is a risk factor for incident Type 2 diabetes [25, 26] and CVD [27]. On the other hand, it has reported that metabolically healthy obesity phenotype has high levels of insulin sensitivity compared to metabolically abnormal obesity phenotype [3-5]. Several studies suggested that the metabolically healthy obesity phenotype had a more favorable distribution of low visceral adipose tissue (VAT), although the total fat mass was similar between metabolically healthy obesity phenotype and metabolically abnormal obesity phenotype [3, 28]. Subcutaneous adipose tissue (SAT) has less facilitative or even protective effects on metabolic abnormalities compared to VAT. In fact, higher VAT/SAT ratio is associated with an increased cardio metabolic risk [29]. Taking these finding together, maintaining MHO phenotype was not associated with higher risk of incident Type 2 diabetes or CVD.

Interestingly, we showed that transitioning from MHO to MANO phenotype was associated with incident CVD. The possible examination is described below. Recent studied have elucidated the impact of so-called sarcopenic obesity $[30,31]$. These studies identified a subset of individuals with non-overweight, in whom the volume of skeletal muscle and its strength decreased [30]. Recent studies showed the possibility that body weight would decrease by the loss of lean mass, including muscle, in spite of fat mass gain in middle and elderly aged individuals $[32,33]$. Besides, the peak of muscle mass was around the age of 45-50 years old and after that, the volume of muscle gradually decreased at an estimated rate of $1-2 \%$ per year [34]. Thus, visceral fat could increase, although body weight decreased. Sarcopenic obesity is associated with hypertension [35] and CVD [36]. In fact, the individuals who transitioning from $\mathrm{MHO}$ to MAO phenotype were older and had higher blood pressure (data not shown). Unfortunately, we did not have data of lean mass and fat mass; however, transitioning from $\mathrm{MHO}$ to MANO might be higher risk of CVD than transitioning from $\mathrm{MHO}$ to MAO.

Strengths of our study include the relatively large number of participants both at baseline and at follow-up. However, this study has some limitations that require consideration. First, because the number of participants who were MANO or MAO phenotype at baseline examination was not enough. In addition, small number of participants were transitioning from $\mathrm{MHO}$ to MANO. However, the main purpose of this study was to investigate the association with maintaining MHO phenotype and incident Type 2 diabetes or CVD. Second, the number of Type 2 diabetes and CVD events is rather small (82 and 51 events respectively). So we cannot deny the possibility of the uncertainty of the outcome. Third, although the average BMI of this study participants was comparable to that of a much larger scale study reported recently from Japan [37], it was far lower BMI than that reported in Western countries [10]. Thus, it is uncertain whether these findings can be generalized in other ethnic groups. Fourth, we cannot deny the possibility of selection bias because our study only included participants who underwent a routine health examination and they might pay more attention to their health than those who do not take such examinations. Fifth, duration of follow-up may have been insufficient to allow us to evaluate the risk of incident CVD. Some clinical outcomes occurred only after a long term follow-up [11]. Thus, further long-term follow-up study is needed. Sixth, we did not have date of dietary exposures and socioeconomic status. Lastly, our analysis is based on the incidence of self-reported CVD. Thus, there is possibility that selection biases could have masked a true association between metabolic phenotypes and CVD.

\section{Conclusion}

Our study showed that maintaining $\mathrm{MHO}$ phenotype was not associated with incident Type 2 diabetes or CVD. On the other hand, transitioning to metabolically abnormal phenotype might lead to incident Type 2 diabetes and CVD for MHO phenotype.

\section{Acknowledgements}

We thank all of the staff members in the Oike Clinic.

\section{References}

[1]. Finucane MM, Stevens GA, Cowan MJ, Danaei G, Lin JK, et al. (2011) National, regional, and global trends in body-mass index since 1980: systematic analysis of health examination surveys and epidemiological studies with 960 country-years and 9・1 million participants. Lancet 377(9765): 557-567.

[2]. Expert Panel on Detection, Evaluation, and Treatment of High Blood Cholesterol in Adults (2001) Executive Summary of The Third Report of The National Cholesterol Education Program (NCEP) Expert Panel on Detection, Evaluation, And Treatment of High Blood Cholesterol In Adults (Adult Treatment Panel III). JAMA 285(19): 2486-2497.

[3]. Brochu M, Tchernof A, Dionne IJ, Sites CK, Eltabbakh GH, et al. (2001) What are the physical characteristics associated with a normal metabolic profile despite a high level of obesity in postmenopausal women? J Clin Endocrinol Metab 86(3): 1020-1025.

[4]. Sims EA (2001) Are there persons who are obese, but metabolically healthy? Metabolism 50(12): 1499-1504.

[5]. Karelis AD, St-Pierre DH, Conus F, Rabasa-Lhoret R, Poehlman ET (2004) Metabolic and body composition factors in subgroups of obesity: what do we know? J Clin Endocrinol Metab 89(6): 2569-2575.

[6]. Karelis AD (2008) Metabolically healthy but obese individuals. Lancet 372(9646): 1281-1283.

[7]. Hashimoto Y, Tanaka M, Okada H, Senmaru T, Hamaguchi M, et al. (2015) Metabolically Healthy Obesity and Risk of Incident CKD. Clin J Am Soc Nephrol 10(4): 578-583.

[8]. Bell JA, Kivimaki M, Hamer M (2014) Metabolically healthy obesity and risk of incident type 2 diabetes: a meta-analysis of prospective cohort studies. Obes Rev 15(6): 504-515.

[9]. Arnlöv J, Sundström J, Ingelsson E, Lind L (2011) Impact of BMI and the metabolic syndrome on the risk of diabetes in middle-aged men. Diabetes Care 34(1): 61-65.

[10]. Aung K, Lorenzo C, Hinojosa MA, Haffner SM (2014) Risk of developing diabetes and cardiovascular disease in metabolically unhealthy normalweight and metabolically healthy obese individuals. J Clin Endocrinol Metab 99(2): 462-468.

[11]. Kramer CK, Zinman B, Retnakaran R (2013) Are metabolically healthy overweight and obesity benign conditions?: A systematic review and metaanalysis. Ann Intern Med 159(11): 758-769.

[12]. Arnlöv J, Ingelsson E, Sundström J, Lind L (2010) Impact of body mass index and the metabolic syndrome on the risk of cardiovascular disease and 
death in middle-aged men. Circulation 121(12): 230-236.

[13]. Bell JA, Hamer M, Sabia S, Singh-Manoux A, Batty GD, et al. (2015) The Natural Course of Healthy Obesity Over 20 Years. J Am Coll Cardiol 65(1): 101-102.

[14]. Khan UI, Wang D, Karvonen-Gutierrez CA, Khalil N, Ylitalo KR, et al. (2014) Progression from metabolically benign to at-risk obesity in perimenopausal women: a longitudinal analysis of study of women across the nation (SWAN). J Clin Endocrinol Metab 99(7): 2516-2525.

[15]. Appleton SL, Seaborn CJ, Visvanathan R, Hill CL, Gill TK, et al. (2013) Diabetes and cardiovascular disease outcomes in the metabolically healthy obese phenotype: a cohort study. Diabetes Care 36(8): 2388-2394.

[16]. Heianza Y, Kato K, Kodama S, Suzuki A, Tanaka S, et al. (2014) Stability and changes in metabolically healthy overweight or obesity and risk of future diabetes: Niigata wellness study. Obesity (Silver Spring) 22(11): 2420-2425.

[17]. Kashiwagi A, Kasuga M, Araki E, Oka Y, Hanafusa T, et al. (2012) International clinical harmonization of glycated hemoglobin in Japan: From Japan Diabetes Society to National Glycohemoglobin Standardization Program values. J Diabetes Investig 3(1): 39-40.

[18]. American Diabetes Association (2010) Diagnosis and classification of diabetes mellitus. Diabetes Care 33(Suppl 1): S62-S69.

[19]. World Health Organization (2000) The Asia-Pacific perspective: Redefining obesity and 424 its treatment. International Diabetes Institute. Health Communications Australia Pty. Ltd. 425 Available at: http://www.wpro.who.int/ nutrition/documents/Redefining obesity/en/

[20]. Anuurad E, Shiwaku K, Nogi A, Kitajima K, Enkhmaa B, et al. (2003) The new BMI criteria for asians by the regional office for the western pacific region of WHO are suitable for screening of overweight to prevent metabolic syndrome in elder Japanese workers. J Occup Health 45(6): 335-343.

[21]. Fukuda T, Hamaguchi M, Kojima T, Hashimoto Y, Ohbora A, et al. (2015) The impact of non-alcoholic fatty liver disease on incident type 2 diabetes mellitus in non-overweight individuals. Liver Int.

[22]. Hsu WC, Araneta MR, Kanaya AM, Chiang JL, Fujimoto W (2015) BMI Cut Points to Identify At-Risk Asian Americans for Type 2 Diabetes Screening. Diabetes Care 38(1): 150-158.

[23]. Alberti KG, Zimmet P, Shaw J (2006) Metabolic syndrome--a new worldwide definition. A Consensus Statement from the International Diabetes Federation. Diabet Med 23(5): 469-480.

[24]. Kahn SE, Hull RL, Utzschneider KM (2006) Mechanisms linking obesity to insulin resistance and type 2 diabetes. Nature 444(7121): 840-846.
[25]. Weinstein AR, Sesso HD, Lee IM, Cook NR, Manson JE, et al. (2004) Relationship of physical activity vs body mass index with type 2 diabetes in women. JAMA 292(10): 1188-1194.

[26]. Wannamethee SG, Shaper AG, Walker M (2005) Overweight and obesity and weight change in middle aged men: impact on cardiovascular disease and diabetes. J Epidemiol Community Health 59(2): 134-139.

[27]. Hubert HB, Feinleib M, McNamara PM, Castelli WP (1983) Obesity as an independent risk factor for cardiovascular disease: a 26-year follow-up of participants in the Framingham Heart Study. Circulation 67(5): 968-977.

[28]. Koster A, Stenholm S, Alley DE, Kim LJ, Simonsick EM, et al. (2010) Body fat distribution and inflammation among obese older adults with and without metabolic syndrome. Obesity (Silver Spring) 18(12): 2354-2361.

[29]. Kaess BM, Pedley A, Massaro JM, Murabito J, Hoffmann U, et al. (2012) The ratio of visceral to subcutaneous fat, a metric of body fat distribution, is a unique correlate of cardiometabolic risk. Diabetologia 55(10): 2622-2630.

[30]. Kohara K (2014) Sarcopenic obesity in aging population: current status and future directions for research. Endocrine 45(1): 15-25.

[31]. Stenholm S, Harris TB, Rantanen T, Visser M, Kritchevsky SB, et al. (2008) Sacopenic obesity: definition, cause and consequences. Curr Opin Clin Nutr Metab Care 11(6): 693-700.

[32]. Bhasin S (2003) Testosterone supplementation for aging-associated sarcopenia. J Gerontol A Biol Sci Med Sci 58(11): 1002-1008.

[33]. Newman AB, Kupelian V, Visser M, Simonsick E, Goodpaster B, et al. (2003) Sarcopenia: alternative definitions and associations with lower extremity function. J Am Geriatr Soc 51(11): 1602-1609.

[34]. Hughes VA, Frontera WR, Roubenoff R, Evans WJ, Singh MA (2002) Longitudinal changes in body composition in older men and women: role of body weight change and physical activity. Am J Clin Nutr 76(2): 473-481.

[35]. Han K, Park YM, Kwon HS, Ko SH, Lee SH, et al. (2014) Sarcopenia as a determinant of blood pressure in older Koreans: findings from the Korea National Health and Nutrition Examination Surveys (KNHANES) 20082010. PLoS One 9(1): e86902.

[36]. Atkins JL, Whincup PH, Morris RW, Lennon LT, Papacosta O, et al. (2014) Sarcopenic obesity and risk of cardiovascular disease and mortality: a population-based cohort study of older men. J Am Geriatr Soc 62(2): 253-260.

[37]. Nakamura Y, Okuda N, Okamura T, Kadota A, Miyagawa N, et al. (2014) Low-carbohydrate diets and cardiovascular and total mortality in Japanese: a 29-year follow-up of NIPPON DATA80. Br J Nutr 112(6): 916-924. 